\title{
Weed Dynamics of Aerobic Rice (Oryza sativa L.) under Chemical and Non-Chemical Weed Management Practices in Irrigated Ecosystem
}

\author{
Himanshu Verma*, S.P. Singh, V.P. Singh, B.S. Mahapatra, \\ Sirazuddin, Neeshu Joshi and Aaradhana Chilwal
}

\author{
Department of Agronomy, Govind Ballabh Pant University of Agriculture and Technology, \\ Pantnagar 263145, Uttarakhand, India \\ *Corresponding author
}

\section{A B S T R A C T}

\begin{tabular}{|l|}
\hline Ke y w or d s \\
Aerobic rice, \\
Sesbania, \\
Pendimethalin, \\
Conoweeder, Weeds. \\
\hline Article Info \\
\hline Accepted: \\
23 October 2017 \\
Available Online: \\
10 December 2017 \\
\hline
\end{tabular}

\begin{abstract}
A field experiment was conducted during kharif season of 2014 at Dr. N. E. Borlaug Crop Research Centre, Pantnagar to assess the effect of different chemical and non-chemical methods of weed control on the weed dynamics of aerobic rice under irrigated ecosystem. Lowest weed density as well as weed dry matter at 25 days stage was found in plots treated with pendimethalin @ $1.25 \mathrm{~kg} / \mathrm{ha}+$ hand weeding (HW) at 25 days after sowing (DAS) followed by wheat mulch straw (4t/ha) followed by (fb) fenoxaprop @ $60 \mathrm{~g} \mathrm{a}$.i/ha. Weed management practices along with pendimethalin @ $1.25 \mathrm{~kg} / \mathrm{ha}$ fb HW (25 DAS) achieved significant control of weeds, high weed control efficiency (WCE) of $93.3 \%$. Among non chemical methods of weed control, Sesbania co-culture fbconoweeder (25 DAS) fb HW (50 DAS) reported WCE (92.3\%).
\end{abstract}

\section{Introduction}

India is having the largest area (43.95 $\mathrm{m} \mathrm{ha})$ under rice in the world and in case of production (106.65 MT), it is next to China, however, productivity of India remains $(2.4$ t/ha) low (Ministry of Agriculture, 2015). Weeds pose a serious threat to the direct seeded rice crop by competing for nutrients, light, space and moisture throughout the growing season. High weed infestation is a major constraint for broader adoption of DSR (Rao et al., 2007). Yield reduction due to weeds is more critical in direct seeded rice than in transplanted rice (Karim et al., 2004). DSR approach demands a weed management strategy that is selective, efficient and cost effective with little or no adverse ecological effects.

Chemical weed control has expanded manifold in DSR and is likely to increase further in response to labour scarcity, rising wages and increased adoption of direct seeding. Unfortunately, indiscriminate use of herbicides is driving the agro-ecosystems towards declining species diversity and, in many situations to herbicide resistance. It was found that continuous use of a single herbicide can produce quantitative changes in weed community composition in just five years (Singh et al., 2009). Presently available 
rice herbicides have narrow spectrum activity and limited efficacy when used alone, and hence rarely provide season long weed control. Variation in weed flora composition and their pattern of emergence during growing season is also key factor influencing level of weed control achieved with herbicides (Khalique et al., 2012 and Kumar et al., 2002). Therefore, present research was carried out to study the effect of chemical and non-chemical weed management practices on weed dynamics of aerobic rice and also its yield potential.

\section{Materials and Methods}

A field experiment was carried out at N.E. Borlaug Crop Research Centre, G.B.P.U.A \& T, Pantnagar during kharif 2014 in a Randomized Block Design with ten treatments and three replications. Ten treatments viz. weedy check; wheat straw mulch (4t/ha) fb one hand weeding (25 DAS); wheat straw mulch (4t/ha) fb fenoxaprop @ $60 \mathrm{~g}$ a.i/ha; Sesbania co-culture $\mathrm{fb}$ conoweeder (25 DAS) fb one hand weeding (50 DAS); pendimethalin @ $1.25 \mathrm{~kg} \mathrm{a.i/ha} \mathrm{fb}$ one hand weeding (25 DAS); pendimethalin @ $1.25 \mathrm{~kg}$ a.i/ha fb conoweeder (25 DAS); Sesbania co-culture fb pendimethalin @ 1.25 kg a.i/ha fb 2, 4-D @ 0.5 kg a.i/ha; bispyribac sodium @ $20 \mathrm{~g}$ a.i/ha; tank mix of fenoxaprop @ $60 \mathrm{~g}$ and 2,4- D@ $0.5 \mathrm{~kg} / \mathrm{ha}$ and weed free are used in experiment. Variety of rice and Sesbania was Sarjoo-52 and Pant Ses-1 respectively. Observations on weed density were recorded at 25, 50, 75, 100 days after sowing (DAS) and at maturity by randomly placing a quadrate of $50 \mathrm{~cm} \times 50 \mathrm{~cm}$ at five places in each plot. The weeds inside each quadrate were uprooted, cleaned and dried. After drying, weight was taken and weed control efficiency was calculated by using the formula,

WCE (\%) ${ }^{\text {WDC - WDT }} 100$
Where,

WDC $=$ Weed dry weight in control plot $\left(\mathrm{g} / \mathrm{m}^{2}\right)$

WDT $=$ Weed dry weight in treated plot $(\mathrm{g} /$ $\mathrm{m}^{2}$ )

\section{Results and Discussion}

Different weed species of the experimental field were collected, identified and classified in to grassy, sedges and broad leaf weeds (BLWs). Thirteen weed species (Grasses- 3, Sedges- 4 and BLWs- 6) were recorded in the experimental field. Among these weed species Echinochloa crus-galli, Echinochloa colona, Leptochloa chinensis, Cyperus rotundus, Cyperus iria, Cyperus difformis and Fimbristylis milliacea were dominant. Similar trend was observed by number of workers Tomar et al., (2002); Kathiresan and Manoharan (2002). The total number of weeds owing to different weed control practices was influenced significantly at all the stages 25, 50, 75, 100 and at maturity stage of rice. Among the various treatments, highest total weed density was found in weedy check followed by Sesbania co-culture fb conoweeder (25 DAS) fb hand weeding (50 DAS) at 25 days stage while at 50, 75, 100 and maturity stage, highest total weed density was recorded in weedy check followed by wheat straw mulch (4 t/ha) fb fenoxaprop @ $60 \mathrm{~g} / \mathrm{ha}$ (Table 1). No weed density was found in weed free plots during all the stages of crop growth. This was in accordance with Singh et al., 2002.

Among treated plots, lowest total weed density at 25 days stage and at maturity, was found in pendimethalin @ $1.5 \mathrm{~kg} / \mathrm{ha} \mathrm{fb} \mathrm{HW}$ (25 DAS) and at 50 days stage, lowest total weed density was recorded in tank mixture of fenoxaprop@60 g /ha and 2, 4- D (38 EC) @ $0.5 \mathrm{~kg} / \mathrm{ha}$. 
Table.1 Effect of treatments on total weed density and total dry matter accumulation by weeds at various stages of crop growth

\begin{tabular}{|c|c|c|c|c|c|c|c|c|c|c|c|}
\hline \multirow{2}{*}{ S.No } & \multirow[b]{2}{*}{ Treatments } & \multicolumn{5}{|c|}{ Total weed density } & \multicolumn{5}{|c|}{ Total dry matter accumulation } \\
\hline & & $\begin{array}{c}25 \\
\text { DAS }\end{array}$ & $\begin{array}{c}50 \\
\text { DAS }\end{array}$ & $\begin{array}{c}75 \\
\text { DAS }\end{array}$ & $\begin{array}{c}100 \\
\text { DAS }\end{array}$ & At maturity & $\begin{array}{c}25 \\
\text { DAS }\end{array}$ & $\begin{array}{c}50 \\
\text { DAS }\end{array}$ & $\begin{array}{c}75 \\
\text { DAS }\end{array}$ & $\begin{array}{c}100 \\
\text { DAS }\end{array}$ & At maturity \\
\hline $\mathrm{T}_{1}$ & Weedy Check & $\begin{array}{c}13.19 \\
(173.0) \\
\end{array}$ & $\begin{array}{c}15.74 \\
(246.7) \\
\end{array}$ & \begin{tabular}{|c|}
13.67 \\
$(186.0)$
\end{tabular} & $\begin{array}{c}13.67 \\
(186.0) \\
\end{array}$ & $\begin{array}{c}12.82 \\
(163.3)\end{array}$ & $\begin{array}{c}5.13 \\
(25.3) \\
\end{array}$ & $\begin{array}{c}37.0 \\
(1365.5)\end{array}$ & $\begin{array}{c}36.8 \\
(1360.0)\end{array}$ & $\begin{array}{c}34.1 \\
(1160.8) \\
\end{array}$ & $\begin{array}{c}33.6 \\
(1126.7) \\
\end{array}$ \\
\hline $\mathrm{T}_{2}$ & WS fb HW (25 DAS) & $\begin{array}{c}9.43 \\
(88.0) \\
\end{array}$ & $\begin{array}{c}9.09 \\
(81.7) \\
\end{array}$ & $\begin{array}{c}8.43 \\
(70.0) \\
\end{array}$ & $\begin{array}{c}7.83 \\
(60.3)\end{array}$ & $\begin{array}{c}7.00 \\
(48.0)\end{array}$ & $\begin{array}{c}4.15 \\
(16.7)\end{array}$ & $\begin{array}{c}10.9 \\
(117.2)\end{array}$ & $\begin{array}{c}25.5 \\
(650.1)\end{array}$ & $\begin{array}{c}25.0 \\
(625.8)\end{array}$ & $\begin{array}{c}22.9 \\
(523.8)\end{array}$ \\
\hline $\mathrm{T}_{3}$ & WS fb FP@60 gai/ha & $\begin{array}{c}5.83 \\
(33.0) \\
\end{array}$ & \begin{tabular}{|c|}
11.93 \\
$(141.3)$ \\
\end{tabular} & \begin{tabular}{|c|}
11.03 \\
$(120.7)$ \\
\end{tabular} & $\begin{array}{c}11.70 \\
(136.0) \\
\end{array}$ & $\begin{array}{c}11.00 \\
(120.0) \\
\end{array}$ & $\begin{array}{c}2.34 \\
(4.53) \\
\end{array}$ & \begin{tabular}{|c|}
10.0 \\
$(99.1)$ \\
\end{tabular} & $\begin{array}{c}17.2 \\
(293.7) \\
\end{array}$ & $\begin{array}{c}19.5 \\
(379.9) \\
\end{array}$ & $\begin{array}{c}18.3 \\
(333.3) \\
\end{array}$ \\
\hline $\mathrm{T}_{4}$ & $\begin{array}{c}\text { Sesfb CW }(25 \\
\text { DAS) fb } \\
\text { HW (50 DAS) }\end{array}$ & $\begin{array}{c}11.27 \\
(126.0)\end{array}$ & $\begin{array}{c}8.62 \\
(73.3)\end{array}$ & $\begin{array}{c}6.03 \\
(35.3)\end{array}$ & $\begin{array}{c}6.73 \\
(44.0)\end{array}$ & $\begin{array}{c}6.22 \\
(37.7)\end{array}$ & $\begin{array}{c}4.53 \\
(19.5)\end{array}$ & $\begin{array}{c}10.3 \\
(105.0)\end{array}$ & $\begin{array}{c}6.5 \\
(41.4)\end{array}$ & $\begin{array}{c}22.2 \\
(490.6)\end{array}$ & $\begin{array}{c}21.4 \\
(455.3)\end{array}$ \\
\hline $\mathrm{T}_{5}$ & $\begin{array}{c}\text { Pendi @ } 1.25 \\
\text { kg/ha fb HW(25 DAS) }\end{array}$ & $\begin{array}{c}5.17 \\
(26.0)\end{array}$ & $\begin{array}{c}7.46 \\
(54.7) \\
\end{array}$ & $\begin{array}{c}6.27 \\
(38.3) \\
\end{array}$ & $\begin{array}{c}6.95 \\
(47.0)\end{array}$ & $\begin{array}{c}6.22 \\
(37.7)\end{array}$ & $\begin{array}{l}1.97 \\
(2.9) \\
\end{array}$ & $\begin{array}{c}9.6 \\
(91.9)\end{array}$ & $\begin{array}{c}12.1 \\
(146.4)\end{array}$ & $\begin{array}{c}13.1 \\
(170.5)\end{array}$ & $\begin{array}{c}11.5 \\
(131.3)\end{array}$ \\
\hline $\mathrm{T}_{6}$ & $\begin{array}{c}\text { Pendi @1.25 } \\
\mathrm{kg} / \mathrm{ha} \mathrm{fb} \mathrm{CW(25} \mathrm{DAS)}\end{array}$ & $\begin{array}{c}8.87 \\
(77.7) \\
\end{array}$ & $\begin{array}{c}8.50 \\
(71.3) \\
\end{array}$ & $\begin{array}{c}6.00 \\
(35.0) \\
\end{array}$ & $\begin{array}{c}5.80 \\
(32.7)\end{array}$ & $\begin{array}{c}5.45 \\
(28.7) \\
\end{array}$ & $\begin{array}{c}3.86 \\
(13.9) \\
\end{array}$ & $\begin{array}{c}7.7 \\
(57.6) \\
\end{array}$ & $\begin{array}{c}21.2 \\
(449.7) \\
\end{array}$ & $\begin{array}{c}19.8 \\
(391.4)\end{array}$ & $\begin{array}{c}19.6 \\
(383.7) \\
\end{array}$ \\
\hline $\mathrm{T}_{7}$ & $\begin{array}{c}\text { SesfbPendi @ } 1.25 \\
\text { kg/ha fb 2,4-D @ } \\
0.5 \mathrm{~kg} \text { ai /ha }\end{array}$ & $\begin{array}{c}6.22 \\
(37.7)\end{array}$ & $\begin{array}{c}9.42 \\
(87.7)\end{array}$ & $\begin{array}{c}8.31 \\
(68.0)\end{array}$ & $\begin{array}{l}7.55 \\
(56.0)\end{array}$ & $\begin{array}{c}6.98 \\
(47.7)\end{array}$ & $\begin{array}{l}3.07 \\
(8.4)\end{array}$ & $\begin{array}{c}18.8 \\
(351.2)\end{array}$ & $\begin{array}{c}22.2 \\
(492.0)\end{array}$ & $\begin{array}{c}24.0 \\
(574.9)\end{array}$ & $\begin{array}{c}22.0 \\
(484.3)\end{array}$ \\
\hline $\mathrm{T}_{8}$ & BS @ $20 \mathrm{~g}$ ai/ha & $\begin{array}{c}10.49 \\
(109.0) \\
\end{array}$ & $\begin{array}{c}9.66 \\
(92.3) \\
\end{array}$ & $\begin{array}{c}9.45 \\
(88.3)\end{array}$ & $\begin{array}{c}8.14 \\
(65.3)\end{array}$ & $\begin{array}{c}7.87 \\
(61.0)\end{array}$ & $\begin{array}{c}6.71 \\
(44.0)\end{array}$ & $\begin{array}{c}16.2 \\
(262.0)\end{array}$ & $\begin{array}{c}23.9 \\
(573.6)\end{array}$ & $\begin{array}{c}24.1 \\
(580.4)\end{array}$ & $\begin{array}{c}22.8 \\
(517.0)\end{array}$ \\
\hline $\mathrm{T}_{9}$ & $\begin{array}{c}\text { Tank mix of FP @ } 60 \mathrm{~g} \text { ai } / \mathrm{ha} \text { and } 2,4- \\
\mathrm{D} @ 0.5 \mathrm{~kg} \mathrm{ai} / \mathrm{ha}\end{array}$ & \begin{tabular}{|c|}
10.65 \\
$(112.3)$ \\
\end{tabular} & $\begin{array}{c}6.38 \\
(39.7) \\
\end{array}$ & \begin{tabular}{|c|}
8.64 \\
$(73.7)$ \\
\end{tabular} & $\begin{array}{c}7.23 \\
(51.3) \\
\end{array}$ & $\begin{array}{c}6.66 \\
(43.3) \\
\end{array}$ & $\begin{array}{c}5.15 \\
(25.6) \\
\end{array}$ & $\begin{array}{c}10.5 \\
(109.3) \\
\end{array}$ & $\begin{array}{c}19.2 \\
(367.0) \\
\end{array}$ & $\begin{array}{c}24.2 \\
(585.4) \\
\end{array}$ & $\begin{array}{c}23.2 \\
(535.7) \\
\end{array}$ \\
\hline $\mathrm{T} 10$ & Weed free & $\begin{array}{c}1.00 \\
(0.00)\end{array}$ & $\begin{array}{c}1.00 \\
(0.00)\end{array}$ & $\begin{array}{c}1.00 \\
(0.00)\end{array}$ & $\begin{array}{c}1.00 \\
(0.00)\end{array}$ & $\begin{array}{c}1.00 \\
(0.00)\end{array}$ & $\begin{array}{c}1.00 \\
(0.00 \\
)\end{array}$ & $\begin{array}{c}1.00 \\
(0.00) \\
\end{array}$ & $\begin{array}{c}1.00 \\
(0.00)\end{array}$ & $\begin{array}{c}1.00 \\
(0.00) \\
\end{array}$ & $\begin{array}{r}1.00 \\
(0.00) \\
\end{array}$ \\
\hline & SEm+ & 0.14 & 0.11 & 0.08 & 0.08 & 0.06 & 0.11 & 0.11 & 0.12 & 0.11 & 0.08 \\
\hline & $\mathrm{CD}$ at $5 \%$ & 0.42 & 0.33 & 0.23 & 0.25 & 0.17 & 0.33 & 0.31 & 0.35 & 0.32 & 0.25 \\
\hline
\end{tabular}

WS- Wheat straw mulch (4 t/ha), FP- Fenoxaprop-p-ethyl (9.3 EC), Pendi- Pendimethalin (30 EC), CW- Conoweeder, HW- Hand weeding, BS- Bispyribac sodium (10 SC), 2,4-D- 2,4 dichlorophenoxy acidic acid (38 EC), Ses- Sesbania co-culture, fb- followed by, DAS-days after sowing. 
Table.2 Effect of treatments on yield and yield attributing characters

\begin{tabular}{|c|c|c|c|c|c|c|c|c|c|c|}
\hline S.No. & Treatments & $\begin{array}{l}\text { No of } \\
\text { panicle } \\
/ \mathrm{m}^{2}\end{array}$ & $\begin{array}{l}\text { Panicle } \\
\text { length }(\mathrm{cm})\end{array}$ & $\begin{array}{l}\text { No of } \\
\text { grains / } \\
\text { panicle }\end{array}$ & $\begin{array}{l}1000- \\
\text { grain } \\
\text { weight }(\mathrm{g})\end{array}$ & $\begin{array}{l}\text { Grain } \\
\text { yield } \\
\mathrm{kg} / \mathrm{ha}\end{array}$ & $\begin{array}{l}\text { Straw } \\
\text { yield } \\
\mathrm{kg} / \mathrm{ha}\end{array}$ & $\begin{array}{l}\text { Grain: } \\
\text { Straw } \\
\text { ratio }\end{array}$ & $\begin{array}{l}\text { biolog } \\
\text { ical } \\
\text { yield }\end{array}$ & $\begin{array}{l}\text { Harves } \\
t \text { Index } \\
\%\end{array}$ \\
\hline $\mathrm{T}_{1}$ & Weedy Check & 14.0 & 25.7 & 63.7 & 20.6 & & & & & \\
\hline $\mathrm{T}_{2}$ & WS fb HW(25 DAS) & 162.0 & 26.3 & 130.0 & 21.6 & 209 & 983 & 0.21 & 1192 & 17.52 \\
\hline $\mathrm{T}_{3}$ & WS fb FP @ 60 g ai/ha & 199.0 & 26.2 & 136.7 & 22.4 & 945 & 6099 & 0.65 & 10043 & 39.27 \\
\hline $\mathrm{T}_{4}$ & $\begin{array}{l}\text { Sesfb CW(25 DAS) fb HW } \\
\text { (50 DAS) }\end{array}$ & 187.3 & 27.3 & 139.3 & 22.5 & 4125 & 5830 & 0.71 & 9955 & 41.43 \\
\hline $\mathrm{T}_{5}$ & $\begin{array}{l}\text { Pendi @ } 1.25 \mathrm{~kg} / \mathrm{ha} \\
\text { fbHW(25 DAS) }\end{array}$ & 302.0 & 26.7 & 125.0 & 24.9 & 4694 & 6495 & 0.72 & 11189 & 41.96 \\
\hline $\mathrm{T}_{6}$ & $\begin{array}{l}\text { Pendi @ } 1.25 \mathrm{~kg} / \mathrm{ha} \mathrm{fb} \\
\mathrm{CW}(25 \text { DAS })\end{array}$ & 223.7 & 26.8 & 134.7 & 23.4 & 5139 & 5992 & 0.86 & 11131 & 46.08 \\
\hline $\mathrm{T}_{7}$ & $\begin{array}{l}\text { SesfbPendi @ } 1.25 \mathrm{~kg} / \mathrm{ha} \mathrm{fb} 2,4-\mathrm{D} \\
@ 0.5 \mathrm{~kg} \text { ai } / \mathrm{ha}\end{array}$ & 222.0 & 26.5 & 109.3 & 21.3 & 4736 & 5809 & 0.81 & 10545 & \begin{tabular}{|l|l}
544.91 \\
\end{tabular} \\
\hline $\mathrm{T}_{8}$ & BS@ 20 g ai/ha & 176.7 & 27.3 & 137.7 & 23.8 & 3417 & 4092 & 0.84 & 7509 & 45.53 \\
\hline $\mathrm{T}_{9}$ & $\begin{array}{l}\text { Tank mix of FP @ } 60 \mathrm{~g} \text { ai/ha and } \\
2,4-\mathrm{D} @ 0.5 \mathrm{~kg} \text { ai } / \mathrm{ha}\end{array}$ & 204.0 & 25.1 & 100.7 & 21.3 & \multicolumn{2}{|c|}{$\begin{array}{l}48335907 \\
\end{array}$} & 0.82 & 10740 & 44.98 \\
\hline $\mathrm{T} 10$ & Weed free & 311.0 & 27.8 & 157.0 & 25.4 & \multicolumn{2}{|c|}{36674588} & 0.80 & 8255 & 44.39 \\
\hline SEm+ & & 1.29 & 0.74 & 1.42 & 0.54 & \multicolumn{2}{|c|}{56956661} & 0.85 & 12355 & 46.16 \\
\hline $\begin{array}{c}\text { CD at } \\
5 \%\end{array}$ & & 3.82 & NS & 4.22 & 1.60 & \multicolumn{2}{|l|}{219} & 0.01 & 472 & 0.20 \\
\hline
\end{tabular}


Fig.1 Effect of weed management practices on weed control efficiency at 50 and 75 days stage of crop growth

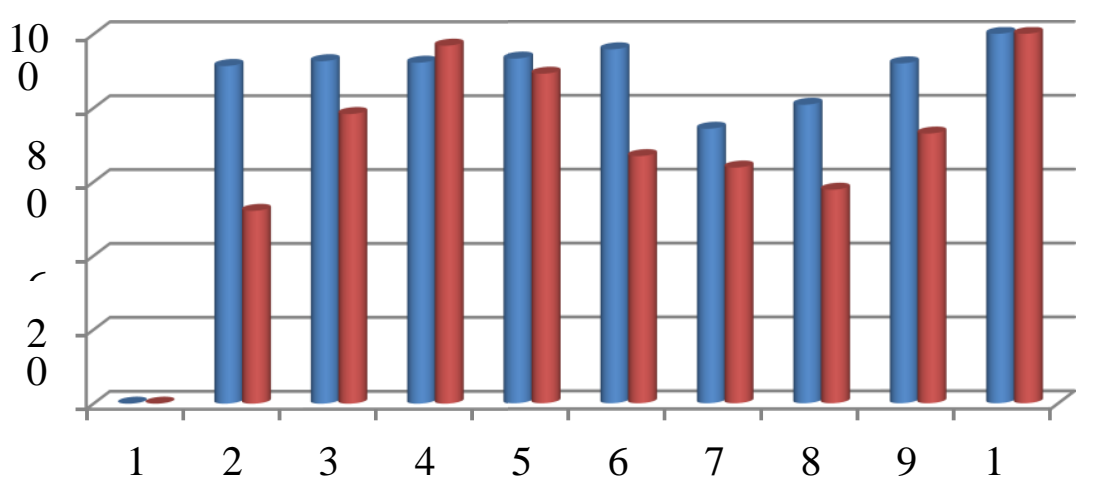

Weed controlEfficiency $(\%)$ 50DAS

1-Weedy, 2- WS fb HW, 3- WS fb FP, 4- Sesfb CW fb HW, 5- Pendifb HW, 6- Pendifb CW, 7- Ses fbPendifb 2,4D, 8- BP, 9- tank mix of FP and 2,4-D, 10 -weed free

This might be due to efficient hand weeding which restrict the growth of weeds in the field of aerobic rice. This was also reported by Khaliq and Matloob (2011) and Khaliq et al., (2012). At 75 and 100 days stage, lowest total weed density was recorded in pendimethalin (30 EC) @ $1.25 \mathrm{~kg} / \mathrm{ha} \mathrm{fb}$ conoweeder (25 DAS) which was statistically at par with Sesbaniaco-culture fb conoweeder (25 DAS) fb HW (50 DAS). Application of different pre emergence herbicides like pendimethalin has been found to control weed satisfactorily in direct seeded rice. This was also in line with Pellern and Webster (2004) and Baloch et al., (2005).

Dry matter accumulation of total weeds varied due to different weed control practices significantly at all the stage of crop growth. Highest total weed dry matter accumulation at 25 days stage of crop growth was recorded in plots treated with bispyribac sodium (10 SC) @ $20 \mathrm{~g}$ /ha while at 50,75, 100 and maturity stage, highest total weed dry matter accumulation was recorded in weedy check (Table 1). Among treatments, lowest total dry matter accumulation at 25, 50,100 and maturity stage was found in plots treated with pendimethalin (30 EC) @ $1.25 \mathrm{~kg} / \mathrm{ha} \mathrm{fb} \mathrm{HW}$
(25 DAS) while at 75 days stage, in plots treated with Sesbania co-culture fbconoweeder (25 DAS) fb hand weeding (50 DAS). This might be due to application of pendimethalin along with one hand weeding done at 25 DAS which effectively reduced the weed growth as well as weed dry matter accumulation in direct -seeded rice. Similar findings were in line with Rao et al., (2008).

Weed control practices had significant effect on WCE at 50 and 75 days stage of the crop growth. 100 percent WCE was recorded in the weed free plots at both the stages while among treated plots, highest WCE of $95.8 \%$ and $96.9 \%$ at 50 DAS and 75 DAS respectively was found in plots treated with pendimethalin (30 EC) @ $1.25 \mathrm{~kg} / \mathrm{ha} \mathrm{fb}$ conoweeder (25 DAS) and Sesbania coculture fb conoweeder (25 DAS) fb HW (50 DAS) respectively (Fig 1).

This might be due to application of preemergence herbicide viz. pendimethalin (30 EC) @ $1.25 \mathrm{~kg} / \mathrm{ha}$ followed by cono weeding at 25 DAS which had great impact against weeds present in plots and recorded highest WCE of $95.8 \%$ at 50 DAS. Similar trend was also observed by (Rao et al., 2008). 
Yield and yield attributing characters

All weed control treatments applied plots produced significantly higher number of panicles/m2, 1000 grain weight, number of grains/panicle, grain yield, straw yield, biological yield, grain: straw ratio and harvest index than weedy check except panicle length which was found non-significant. The highest number of panicles $/ \mathrm{m}^{2}, 1000$ grain weight, grain yield and grain: straw ratio was obtained in weed free treatment which was statistically at par with pendimethalin (30 EC) @ 1.25 $\mathrm{kg} / \mathrm{ha} \mathrm{fb}$ hand weeding (25 DAS) and also highest number of grains/panicle and biological yield were obtained in weed free treatment which was statistically at par with Sesbania co-culture fb conoweeder (25 DAS) $\mathrm{fb}$ hand weeding (50 DAS) (Table 2). This might be due to effectiveness of hand weeding at 25 DAS which effectively controlled the weeds but has been restricted due to several economical and technological factors. Similar findings were in line with Khaliq and Matloob (2011), Dixit and Bhan (2001). High yield of rice due to application of pre emergence herbicide pendimethalin (30 EC) @ $1.25 \mathrm{~kg} / \mathrm{ha} \mathrm{fb}$ hand weeding (25 DAS) might be due to inhibition of weed dry weight as well as reduced crop weed competition, ultimately increased the grain yield. Similar results were given by Singh et al., (2005).

\section{References}

Baloch MS, Hassan G and Morimoto $\mathrm{T}$ (2005). Weeding techniques in transplanted and wet seeded rice in Pakistan. Weed Biological Management. 5: 190-196.

Dixit A and Bhan VM (2001).Weed Management in Cereals. Pesticides Information. 27: 4-8.

Karim SMR, Man AB and Sahid IB (2004). Weed problems and their management in rice fields of Malaysia: An overview.
Weed Biological Management. 4: 177186.

Kathiresan G and Manoharan ML (2002). Effect of seed rate and methods of weed control on weed growth and yield of direct sown rice. Indian Journal of Agronomy. 47(2): 212-215.

Khaliq A and Matloob A (2011). Weed crop competition period in three fine rice cultivars under direct seeded rice culture. Pakistan Journal of Weed Science Research. 17(3): 229-243.

Khaliq A, Matloob A, Ahmad N, Rasul F and Awan IU (2012). Post emergence chemical weed control in direct seeded fine rice. The Journal of. Animal and Plant Sciences. 22(4): 1101-1106.

Kumar P, Jha D, Kumar A, Chaudhary MK, Grover RK, Singh RK, Mitra A, Joshi PK, Singh A, Badal PS, Mittal S and Ali J (2002). Economic analysis of total factor productivity of crop sector in Indo-Gangetic Plain of India by district and region. Agricultural Economics Research Report 2. New Delhi, India: Indian Agricultural Research Institute.

Ministry of Agriculture. (2015) www.agricoop.com

Pellerin KJ and Webster EP (2004). Imazethapyr at different rates and timings in drill and water seeded imidazolinone-tolerant rice. Weed Technology. 18(2): 223-227.

Rao AN, Johnson DE, Sivaprasad B, Ladha JK and Mortimer AM (2007). Weed management in direct-seeded rice. Advances in Agronomy. 93: 153-255.

Rao AS, Ratnam M and Reddy TY (2008). Weed Management in Direct-seeded Semi Dry Rice. Indian Journal of Weed Science. 40 (3 \& 4): 153-156.

Singh G, Singh OP, Kumar J, Mehta RK, Kumar V and Singh PP (2005). Effect of weed management practices on direct seeded rice (Oryza sativa) under puddled low lands. Indian Journal of 
Agronomy. 50: 35-37.

Singh RK, Sharma SN, Singh R and Pandey MD (2002). Efficacy of method of planting and weed control measures on nutrient removal of rice (Oryza sativa L.) and associated weeds. Crop Research.24 (3):425-429.

Singh S, Chhokar RS, Gopal R, Ladha, JK, Gupta, RK, Kumar V and Singh $M$ (2009). Integrated weed management: A key to success for direct-seeded rice in the Indo-Gangetic Plains. In "Integrated
Crop and Resource Management in the Rice-Wheat System of South Asia." Ladha J.K, Singh Y, Erenstein O, Hardy B (Eds). International Rice Research Institute, Los Banos, Philippines pp 261- 278 .

Tomar TPS, Rana NS, Kumar S and Singh R (2002). Effect of integrated Weed Management on Upland Direct-Seeded Rice and Associated Weeds. Annals of Agricultural Research. 23(3): 423-429.

\section{How to cite this article:}

Himanshu Verma, S.P. Singh, V.P. Singh, B.S. Mahapatra, Sirazuddin, Neeshu Joshi and Aaradhana Chilwal. 2017. Weed Dynamics of Aerobic Rice (Oryza sativa L.) under Chemical and Non-Chemical Weed Management Practices in Irrigated Ecosystem. Int.J.Curr.Microbiol.App.Sci. 6(12): 3159-3165. doi: https://doi.org/10.20546/ijcmas.2017.612.369 